\title{
Spin-orbit coupling induced by effective mass gradient
}

\author{
A. Matos-Abiague \\ Institute for Theoretical Physics, University of Regensburg, 93040 Regensburg, Germany \\ (Received 7 January 2010; revised manuscript received 4 March 2010; published 9 April 2010)
}

\begin{abstract}
The existence of a spin-orbit coupling (SOC) induced by the gradient of the effective mass in lowdimensional heterostructures is revealed. In structurally asymmetric quasi-two-dimensional semiconductor heterostructures the presence of a mass gradient across the interfaces results in a SOC which competes with the SOC created by the electric field in the valence band. However, in graded quantum wells subjected to an external electric field, the mass-gradient-induced SOC can be finite even when the electric field in the valence band vanishes.
\end{abstract}

DOI: 10.1103/PhysRevB.81.165309

PACS number(s): 71.70.Ej, 73.61.Ey, 73.20.Qt, 73.21.Fg

Semiconductor spintronics is an emerging field based on the controlled manipulation of the carrier spins for data processing and device operations. ${ }^{1,2}$ Most proposals for spintronic devices rely on the ability of manipulating electron spins by using the spin-orbit coupling (SOC), which is the most fundamental spin-dependent interaction in nonmagnetic semiconductors. ${ }^{2}$ In semiconductor heterostructures the SOC results from the lack of inversion symmetry. The bulk inversion asymmetry of zinc-blende semiconductors leads to the so-called Dresselhaus SOC (Ref. 3) while the structure inversion asymmetry (SIA) of the heterostructure itself results in the Bychkov-Rashba (BR) SOC. ${ }^{4}$

In this paper I focus on the investigation of the BR-type SOC and show that, in addition to the SOC generated by the electric field in the valence band, ${ }^{5-7}$ there is a mass-gradient contribution to the SIA-induced SOC, which, although implicitly contained in some previous calculations, ${ }^{2,5-8}$ has not been recognized till now. In general, the two contributions compete. However, in some specific cases the mass-gradientinduced SOC dominates. It is expected to be particularly relevant in surfaces and metal/semiconductors interfaces, where the effective mass has strong and sharp changes. The identification of the mass-gradient contribution not only clarifies the nature and origin of the SIA-induced SOC but could also lead to new control mechanisms for spin manipulation in heterostructures.

The emergence of SOC due to the existence of a mass gradient can be better understood by establishing an analogy between the nonrelativistic limit of Dirac's theory and the effective-mass Hamiltonian for conduction electrons in semiconductor heterostructures. For simplicity and without loss of generality, I consider the case of a time-independent system in the presence of an electrostatic potential $V$. Starting with the time-independent Dirac equation the upper $\psi_{u}$ and lower $\psi_{l}$ components of the four-component spinor $\Psi$ $=\left(\psi_{u}, \psi_{l}\right)^{T}$ are found to be coupled through the equations ${ }^{9}$

$$
\begin{gathered}
(\boldsymbol{\sigma} \cdot \mathbf{p}) \psi_{l}=\frac{1}{c}(\epsilon-V) \psi_{u}, \\
(\boldsymbol{\sigma} \cdot \mathbf{p}) \psi_{u}=\frac{1}{c}\left(\epsilon-V+2 m_{0} c^{2}\right) \psi_{l},
\end{gathered}
$$

where $\epsilon$ is the particle energy (measured from the rest energy $\left.m_{0} c^{2}\right), \boldsymbol{\sigma}$ is the vector of Pauli matrices and $\mathbf{p}, m_{0}$, and $c$, refer to the momentum operator, the bare electron mass, and the velocity of light, respectively. It follows from Eq. (2) that $\psi_{l}$ is smaller than $\psi_{u}$ by a factor $\sim v / c$. Thus, in the nonrelativistic limit $(v \ll c)$ the main contribution to the fourcomponent spinor comes from $\psi_{u}$. Using Eq. (2) one can eliminate $\psi_{l}$ from Eq. (2) and obtain, after some algebra, an equation that involves only $\psi_{u}$,

$$
\left\{\mathbf{p} \cdot\left(\frac{1}{2 \mu} \mathbf{p}\right)+\frac{\hbar}{2}\left[\boldsymbol{\nabla}\left(\frac{1}{\mu}\right) \times \mathbf{p}\right] \cdot \boldsymbol{\sigma}+V\right\} \psi_{u}=\epsilon \psi_{u},
$$

where I have introduced the position-dependent potential mass, $\mu=m_{0}\left[1+(\epsilon-V) / 2 m_{0} c^{2}\right]$. Note that no approximation has been made in deriving Eq. (3). The two-component spinor $\psi_{u}$ is, however, not normalized. The standard procedure to overcome this problem in the nonrelativistic limit is to introduce a new normalized two-component spinor $\tilde{\psi}$ $=\left(1+p^{2} / 8 m_{0} c^{2}\right) \psi_{u} \cdot{ }^{9}$ In doing this, however, some approximations has to be made and the Hamiltonian for the normalized spinor $\tilde{\psi}$ acquires additional terms. ${ }^{9}$ However, these additional terms are irrelevant for our discussion here and will be omitted in our analysis.

The Hamiltonian in Eq. (3) is Hermitian and resembles the effective-mass Hamiltonian describing the motion of electrons in a solid with position-dependent effective mass. Interestingly, the spin-orbit coupling seems to originate from the gradient of the potential mass $\mu$. In the Dirac theory the energy gap, $E_{0}=2 m_{0} c^{2}$, which separates the energy spectra of the free particles and antiparticles is a position-independent constant. Therefore, the only position dependence in $\mu$ which can lead to a finite SOC has to come from the electrostatic potential $V$. Thus, in the Dirac theory, the SOC emerges purely from the electric field $\mathbf{E}=(-1 / e) \boldsymbol{\nabla} V$ (here $e$ denotes the electron charge). In the nonrelativistic approximation the dominant energy is the vacuum gap $E_{0}$ and the inverse potential mass can be approximated as $\mu^{-1} \approx m_{0}^{-1}$ $+(V-\epsilon) / 2 m_{0}^{2} c^{2}$. As a result the SOC reduces to the wellknown form ${ }^{9}$

$$
H_{\mathrm{so}}=\frac{\hbar}{2}\left[\boldsymbol{\nabla}\left(\frac{1}{\mu}\right) \times \mathbf{p}\right] \cdot \boldsymbol{\sigma} \approx \frac{\hbar c^{2}}{E_{0}^{2}}(\nabla V \times \mathbf{p}) \cdot \boldsymbol{\sigma} .
$$

In the case of semiconductors the analog to the potential mass $\mu$ is the effective mass $m^{*}$ while the equivalent to the 


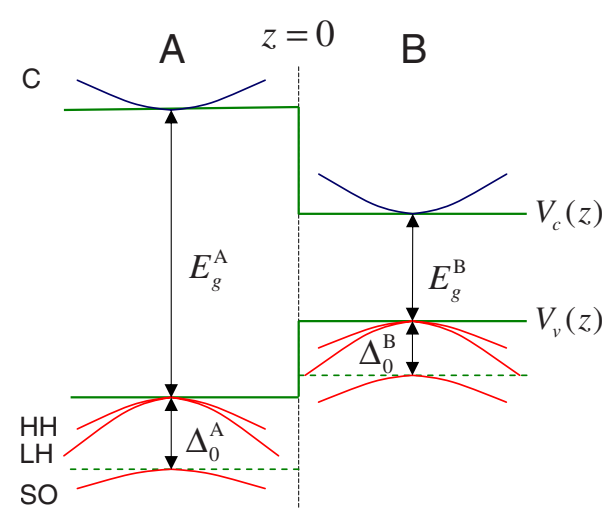

FIG. 1. (Color online) Schematic of the band-edge profile of an $\mathrm{A} / \mathrm{B}$ semiconductor interface located at $z=0$. The conduction, heavy hole, light hole, and split-off bands are labeled as $\mathrm{C}, \mathrm{HH}, \mathrm{LH}$, and $\mathrm{SO}$, respectively.

vacuum gap $E_{0}$ is the energy gap $E_{g}$ separating the energy spectrum of electrons in the conduction band from the hole spectrum in the valence band. In contrast to the vacuum, where $E_{0}$ is a constant, in semiconductor heterostructures the energy gap $E_{g}$ becomes position dependent. Therefore, the position dependence of the effective mass may originate from both the electrostatic potential $V$ and the band gap $E_{g}$. As a result, in addition to the conventional SOC produced purely by the electric field a finite SOC contribution induced by the position dependence of the effective mass emerges.

To investigate in more details the mass-gradient-induced SOC, I consider a semiconductor heterostructure grown in the $z$ direction. In such a case the mass-gradient-induced SOC can be related to the well-known BR SOC observed in quasi-two-dimensional (2D) systems with SIA. ${ }^{4}$

The effective Hamiltonian describing the motion of the conduction-band electrons in the heterostructure can be obtained by using the envelope function approximation. I consider the $(8 \times 8)$ Kane Hamiltonian ${ }^{2,6,10}$ which accounts for the $\Gamma_{6 c}, \Gamma_{8 v}$, and $\Gamma_{7 v}$ bands (see bands $\mathrm{C}, \mathrm{HH}$ and LL, and SO bands, respectively, in Fig. 1). The conduction- and valence-band states can be decoupled by using the foldingdown (Löwdin) technique. ${ }^{2,6,11}$ Neglecting the nonparabolicity effects, the effective Hamiltonian for the conduction electrons is found to be $\mathrm{e}^{2,5,8}$

$$
H_{\mathrm{eff}}=\frac{p_{\|}^{2}}{2 m^{*}(z)}-\frac{\hbar^{2}}{2} \frac{d}{d z}\left[\frac{1}{m^{*}(z)} \frac{d}{d z}\right]+V_{c}(z)+H_{\mathrm{so}},
$$

where

$$
\frac{1}{m^{*}(z)}=\frac{1}{m_{0}}-\frac{2 \widetilde{P}^{2}}{3 m_{0}^{2}}\left[\frac{2}{V_{v}(z)}+\frac{1}{V_{v}(z)-\Delta_{0}(z)}\right]
$$

is the $z$-dependent, inverse effective mass for the conduction band electrons and

$$
H_{\text {so }}=\frac{\alpha(z)}{\hbar}\left(p_{y} \sigma_{x}-p_{x} \sigma_{y}\right)
$$

with

$$
\alpha(z)=\frac{\hbar^{2}}{3 m_{0}^{2}} \frac{d}{d z}\left[\frac{\tilde{P}^{2}}{V_{v}(z)}-\frac{\tilde{P}^{2}}{V_{v}(z)-\Delta_{0}(z)}\right]
$$

is the SIA-induced SOC. In the equations above $p_{x}$ and $p_{y}$ are the components of the in-plane momentum $\mathbf{p}_{\|}$and $\widetilde{P}$ $=\left\langle S\left|p_{x}\right| P\right\rangle$ represents the nonvanishing momentum matrix elements involving the $s$-like band edge Bloch state $(|S\rangle)$ of the conduction band and the $p$-like hole states $(|P\rangle$ $=|X\rangle,|Y\rangle,|Z\rangle)$. The correction to the effective mass due to the interaction with remote bands can be included by using perturbation theory. ${ }^{6,12}$ As shown in Fig. $1, \Delta_{0}(z)$ refers to the spin-orbit splitting energy while $V_{c}(z)$ and $V_{v}(z)$ are the potential profiles of the conduction- and valence-band edges, respectively.

For the case of a quantum well grown along the $z$ direction one can obtain an effective Hamiltonian describing the in-plane motion of a two-dimensional electron gas by averaging Eq. (5) with the spin-independent $z$ component, $f(z)$, of the wave function. This results in the so-called BR SOC with $\alpha_{\mathrm{BR}}=\langle\alpha(z)\rangle_{c}=\int \alpha(z)|f(z)|^{2} d z$ as the BR SOC strength.

At first glance it seems that the BR spin splitting should be proportional to the electric field which breaks the spatial inversion symmetry. However, the fact that for quasi-2D systems with position-independent effective mass the electric field along the direction of confinement must average to zero $^{13}$ (this follows from Ehrenfest's theorem which states that the average force on a bound state vanishes ${ }^{14}$ ) generated intensive discussions about the nature of the electric field causing the BR SOC. ${ }^{5,13,15,16}$ It was later found that for a quantum-well growth along the $z$ direction, with $z$-dependent effective mass, the average electric field $E_{c}$ $\sim\left\langle p_{z}\left[\left(\partial_{z} m^{-1}\right) p_{z}\right]\right\rangle_{c}$ may not vanish. ${ }^{17}$ However, the estimated value for this field was found to be too small ${ }^{16,17}$ as to explain the experimentally observed spin splitting due to the SIA SOC. Actually, the SOC induced by $E_{c}$ represents a high-order correction which is not even present in the effective Hamiltonian obtained with the standard $(8 \times 8)$ Kane approximation.

In an attempt to clarify the origin of the BR SOC, Lassnig $^{5}$ showed that the BR spin splitting in the conduction band is related to the electric field in the valence band $\left[\mathbf{E}_{v}\right.$ $\left.=(-1 / e) \boldsymbol{\nabla} V_{v}\right]$ whose average (over the conduction states) does not necessarily vanish (note that in this case Ehrenfest's theorem does not apply $\left.{ }^{6,7}\right)$. Below I show that in addition to the SOC induced purely by $\mathbf{E}_{v}$ a contribution originating from the existence of a mass gradient appears. I remark, however, that the mass-gradient contribution discussed here is much larger than the one generated by the mass-gradientinduced electric field $E_{c}$ (see discussion in the previous paragraph) and appears readily in the effective Hamiltonian resulting from the $(8 \times 8)$ Kane model. In fact, the BR SOC can be reinterpreted as resulting from the competition between the here-proposed mass-gradient SOC and the SOC induced purely by $\mathbf{E}_{v}$. Interestingly, the mass-gradient contribution (and therefore the BR SOC) can be finite even when the electric field in the valence band vanishes (i.e., $\mathbf{E}_{v}=0$ ). 
Combining Eqs. (6) and (8) one can rewrite the SOC parameter as

$$
\alpha(z)=\frac{\hbar^{2}}{2} \frac{d}{d z}\left[\frac{1}{m^{*}(z)}\right]+\frac{\hbar^{2}}{m_{0}^{2}} \frac{d}{d z}\left[\frac{\tilde{P}^{2}}{V_{v}(z)}\right] .
$$

When modeling semiconductor heterostructures, the momentum matrix element, $\widetilde{P}$, is commonly considered to be position independent. ${ }^{6}$ Within this approximation and taking into account that for most semiconductors $V_{v}$ is of the same order of the band gap (i.e., $\left|V_{v}-E_{g}\right| \ll E_{g}$, one can approximate Eq. (9) as $^{18}$

$$
\alpha(z)=\frac{\hbar^{2}}{2} \frac{d}{d z}\left[\frac{1}{m^{*}(z)}\right]-\frac{\hbar^{2}\left(\widetilde{P} / m_{0}\right)^{2}}{E_{g}^{2}} \frac{d}{d z} V_{v}(z) .
$$

Thus, one can rewrite the SOC in Eq. (7) as $H_{\mathrm{so}}=H_{\mathrm{so}}^{m}+H_{\mathrm{so}}^{v}$, where

$$
H_{\mathrm{so}}^{m}=\frac{\hbar}{2}\left[\boldsymbol{\nabla}\left(\frac{1}{m^{*}}\right) \times \mathbf{p}\right] \cdot \boldsymbol{\sigma}
$$

is the mass-gradient-induced SOC and

$$
H_{\mathrm{so}}^{v}=-\frac{\hbar\left(\widetilde{P} / m_{0}\right)^{2}}{E_{g}^{2}}\left(\boldsymbol{\nabla} V_{v} \times \mathbf{p}\right) \cdot \boldsymbol{\sigma}
$$

is the SOC created by the electric field in the valence band.

Equation (12) has the same structure as Eq. (4), ${ }^{18}$ which confirms that, indeed, $H_{\mathrm{so}}^{v}$ corresponds to the standard SOC generated, purely, by an electric field; in this case by the valence-band electric field $\mathbf{E}_{v}=(-1 / e) \boldsymbol{\nabla} V_{v}$. Consequently, $H_{\mathrm{so}}^{v}$ vanishes when $\mathbf{E}_{v}=0$. On the contrary, it is clear from Eqs. (6) and (11) that even in the case of vanishing $\mathbf{E}_{v}$, the mass-gradient-induced SOC, $H_{\mathrm{so}}^{m}$, remains, in general, finite.

The SOC strength in semiconductor heterostructures is usually evaluated ${ }^{2,5-8}$ by using Eq. (8), which already contains, implicitly, the mass-gradient contribution [note that Eqs. (8) and (10) are equivalent]. However, since the physical interpretation of Eq. (8) is obscure, the mass-gradient contribution has not been recognized till now. By rewriting Eq. (8) in the form of Eq. (10), the physical meaning of each of the contributions to the total SOC become apparent. The mass-gradient-induced SOC emerges then naturally, at the same level as the SOC generated by the valence-band electric field.

For an estimation of the strengths of the mass-gradient and valence-band electric field-induced SOCs, I consider the case of an A/B abrupt interface between two III-V semiconductors (see Fig. 1). In such a case the band parameters, and therefore $m^{*}$ and $V_{v}$, are steplike functions of $z$ and the massgradient and valence-band electric field-induced SOCs in Eq. (9) reduce to

$$
H_{\mathrm{so}}^{m, v}=\frac{\alpha_{m, v}}{\hbar} \delta(z)\left(p_{y} \sigma_{x}-p_{x} \sigma_{y}\right)
$$

with
TABLE I. Interface spin-orbit coupling parameters (in eV $\AA^{2}$ ) for $\mathrm{A} / \mathrm{B}$ abrupt interfaces containing arsenides. $\alpha_{m}$ and $\alpha_{v}$ correspond to the contributions due to the mass gradient and valenceband electric field, respectively, while $\alpha_{\text {int }}$ is the total interface SOC strength.

\begin{tabular}{lcccc}
\hline \hline $\mathrm{A}$ & $\mathrm{B}$ & $\alpha_{m}$ & $\alpha_{v}$ & $\alpha_{\text {int }}$ \\
\hline $\mathrm{GaAs}$ & $\mathrm{AlAs}$ & -35.88 & 39.07 & 3.19 \\
$\mathrm{Ga}_{0.47} \mathrm{In}_{0.53} \mathrm{As}$ & $\mathrm{Al}_{0.48} \mathrm{In}_{0.52} \mathrm{As}$ & -33.39 & 62.09 & 28.7 \\
$\mathrm{Ga}_{0.47} \mathrm{In}_{0.53} \mathrm{As}$ & $\mathrm{InP}$ & -40.68 & 62.73 & 22.05 \\
\hline \hline
\end{tabular}

$$
\alpha_{m}=\frac{\hbar^{2}}{2}\left(\frac{1}{m_{\mathrm{B}}^{*}}-\frac{1}{m_{\mathrm{A}}^{*}}\right)
$$

and

$$
\alpha_{v}=\frac{\hbar^{2}}{m_{0}^{2}}\left(\frac{\widetilde{P}_{\mathrm{A}}^{2}}{E_{g}^{\mathrm{A}}}-\frac{\widetilde{P}_{\mathrm{B}}^{2}}{E_{g}^{\mathrm{B}}}\right),
$$

respectively. Here the values of the parameters in regions A and $\mathrm{B}$ are indicated with the respective labels. Note also, that for a better accuracy, the steplike position dependence of the momentum matrix element has also been considered in Eq. (15).

The calculated values of $\alpha_{m}, \alpha_{v}$, and the total interface SOC strength, $\alpha_{\text {int }}=\alpha_{m}+\alpha_{v}$, for some, experimentally relevant, ${ }^{19-21}$ interfaces are listed in Table I. For all the considered interfaces, $\alpha_{m}$ and $\alpha_{v}$ are of the same order but with opposite signs. Thus, the competition between the massgradient and valence-band electric field-induced SOC contributions results in the overall decrease in the total interface SOC.

In systems in which $\alpha_{m}$ and $\alpha_{v}$ are of the same order (as the ones considered above) the mass-gradient contribution to the SOC is masked by the SOC induced by the valence-band electric field. Therefore the experimental measurement of $\alpha_{m}$ alone may be difficult in such systems. To overcome this problem, I propose to measure $\alpha_{m}$ in a graded, semiconductor quantum well subjected to an external electric field.

For illustration, I consider a $\mathrm{Ga}_{1-x} \mathrm{Al}_{x} \mathrm{As}$-based quantum well with high-potential barriers so that interface effects play a little role and can be neglected. In a linearly graded quantum well (i.e, with $\mathrm{Al}$ concentration varying linearly with the position) the energy gap together with the band parameters become position dependent. For small grading, the band parameters interpolate linearly with the $\mathrm{Al}$ concentration. Consequently, both the potential profile of the conduction $\left(V_{c}^{0}\right)$ and valence $\left(V_{v}^{0}\right)$ band edges change linearly with $z$ [see Fig. 2(a)]. In the presence of a constant external electric field, $\mathbf{E}_{\text {ext }}$, oriented along the growth direction the band-edge profiles are modified as $V_{c}=V_{c}^{0}(z)-e E_{\text {ext }} z$ and $V_{v}=V_{v}^{0}(z)$ $-e E_{e x t} z$. Since $V_{v}^{0}(z)$ is a linear function of $z$ one can find a target electric field for which $V_{v}$ becomes position independent and the total electric field in the valence bands vanishes [see Fig. 2(b)]. Therefore, for such a target external field, $\alpha_{v} \approx 0$, while $\alpha_{m}$ remains finite. Under this condition, the SOC is determined solely by the mass-gradient contribution. 
(a)

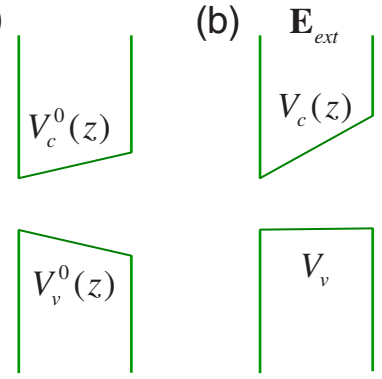

FIG. 2. (Color online) Schematics of the conduction- and valence-band potentials in a linearly graded quantum well with infinite barriers, in the (a) absence and (b) presence of an external electric field $\mathbf{E}_{\text {ext }}$. The external electric field compensates the electric field in the valence band in such a way that the total electric field in the valence band vanishes (i.e., $\boldsymbol{\nabla} V_{v}=0$ ).

I now estimate the values of $\bar{\alpha}_{m}$ (the bar sign stands for position average) and the target external field $E_{\text {target }}$ for a quantum well with $\mathrm{Al}$ concentration varying linearly from $x_{\min }=0$ to $x_{\max }=0.1$, i.e.,

$$
x(z)=x_{\max } z / d .
$$

Here $d=200 \AA$ is the well width. The composition dependence of the band parameters is evaluated according to the interpolation scheme developed in Ref. 12. I then expand Eq. (6) up to the linear order in $x$ [which for the small concentrations considered here $(x \leq 0.1)$ suffices] and obtain the position dependence of the inverse effective mass by using Eq. (16). For the target external field $E_{\text {target }} \approx 30 \mathrm{kV} / \mathrm{cm}$ the valence-band electric field vanishes (i.e., $\bar{\alpha}_{v}=0$ ) while the strength of the mass-gradient-induced SOC is found to be $\bar{\alpha}_{m} \approx-8.74 \mathrm{meV} \AA$. Apart from the sign, this value is of the same order but still larger than the SIA SOC parameters experimentally measured in GaAs-AlGaAs asymmetric quantum wells. ${ }^{19,20}$

Beyond the case of semiconductor heterostructures, the mass-gradient-induced SOC is expected to be relevant in surfaces and metal/semiconductor interfaces across which the values of the effective mass have large and abrupt changes. For example, the mass-gradient contribution to the SOC in the $L$-gap surface state of $\mathrm{Au}(111)$ can be easily estimated by using Eqs. (13) and (14), and the experimental values $m^{*}$ $=0.25 m_{0}$ in $\operatorname{gold}^{22}\left(m^{*}=m_{0}\right.$ in vacuum $)$. Assuming that the surface state is localized within the five outermost surface layers (the potential of the sixth surface layer already shows bulk behavior ${ }^{22}$ ) one obtains $\bar{\alpha}_{m} \approx 323 \mathrm{meV} \AA$, which is already close to the value $396 \mathrm{meV} \AA$ extracted from experiments. ${ }^{22}$ This suggests that the mass-gradient SOC gives the dominant contribution in such a system. ${ }^{23}$

In summary, the existence of a mass-gradient contribution to the SOC in systems with structure inversion asymmetry is revealed. This contribution can be of the same order as the SOC generated by the electric field in the valence band of semiconductors. However, in the particular case of a linearly graded semiconductor quantum well subjected to a conveniently designed external field, the electric field in the valence band vanishes and the remaining SOC is purely induced by the mass gradient. The relevance of the massgradient SOC for surfaces and metal/semiconductor interfaces is briefly addressed.

I am grateful to J. Fabian for fruitful discussions. This work was supported by the Deutsche Forschungsgemeinschaft under Grant No. SFB 689.
${ }^{1}$ I. Žutić, J. Fabian, and S. Das Sarma, Rev. Mod. Phys. 76, 323 (2004).

${ }^{2}$ J. Fabian, A. Matos-Abiague, C. Ertler, P. Stano, and I. Žutić, Acta Phys. Slov. 57, 565 (2007).

${ }^{3}$ G. Dresselhaus, Phys. Rev. 100, 580 (1955).

${ }^{4}$ Y. A. Bychkov and E. I. Rashba, J. Phys. C 17, 6039 (1984).

${ }^{5}$ R. Lassnig, Phys. Rev. B 31, 8076 (1985).

${ }^{6} \mathrm{R}$. Winkler, Spin-Orbit Coupling Effects in Two-Dimensional Electron and Hole Systems (Springer, Berlin, 2003).

${ }^{7}$ R. Winkler, Physica E 22, 450 (2004).

${ }^{8}$ E. A. de Andrada e Silva, G. C. La Rocca, and F. Bassani, Phys. Rev. B 55, 16293 (1997).

${ }^{9}$ J. J. Sakurai, Advanced Quantum Mechanics (Addison-Wesley, Reading, Massachusetts, 1967).

${ }^{10}$ E. O. Kane, Lect. Notes Phys. 133, 13 (1980).

${ }^{11}$ P.-O. Löwdin, J. Chem. Phys. 19, 1396 (1951).

${ }^{12}$ I. Vurgaftman, J. R. Meyer, and L. R. Ram-Mohan, J. Appl. Phys. 89, 5815 (2001).

${ }^{13}$ A. Därr, J. P. Kotthaus, and T. Ando, in Proceedings of the 13th International Conference of Semiconductors, edited by F. G. Fumi (North-Holland, Amsterdam, 1976), p. 774.

${ }^{14}$ J. J. Sakurai, Modern Quantum Mechanics (Addison-Wesley,
Reading, Massachusetts, 1994).

${ }^{15}$ F. J. Ohkawa and Y. Uemura, J. Phys. Soc. Jpn. 37, 1325 (1974).

${ }^{16}$ F. Malcher, G. Lommer, and U. Rössler, Superlattices Microstruct. 2, 267 (1986).

${ }^{17}$ W. Zawadzki and P. Pfeffer, Phys. Rev. B 64, 235313 (2001).

${ }^{18}$ In general, the position dependence of $\widetilde{P}$ leads to an extra contribution in Eq. (10) which is proportional to $d \tilde{P} / d z$. This contribution is absent in the nonrelativistic limit of Dirac's theory, where $\widetilde{P}$ plays the role of $m_{0} c$ (see Ref. 7), which is a positionindependent quantity.

${ }^{19}$ B. Jusserand, D. Richards, G. Allan, C. Priester, and B. Etienne, Phys. Rev. B 51, 4707 (1995).

${ }^{20}$ J. B. Miller, D. M. Zumbühl, C. M. Marcus, Y. B. LyandaGeller, D. Goldhaber-Gordon, K. Campman, and A. C. Gossard, Phys. Rev. Lett. 90, 076807 (2003).

${ }^{21}$ G. Engels, J. Lange, Th. Schäpers, and H. Lüth, Phys. Rev. B 55, R1958 (1997).

${ }^{22}$ J. Henk, M. Hoesch, J. Osterwalder, and A. Ernst, J. Phys.: Condens. Matter 16, 7581 (2004).

${ }^{23}$ Here Eherenfest's theorem applies and the contribution of the average (over the surface states) surface electrostatic field to the SIA SOC must be small. 